\title{
A Mathematical Model for the Control of Cholera Epidemic without Natural Recovery
}

\author{
Olaniyi S. Maliki, Ogbonna C. Chibuike \\ Department of Mathematics, Michael Okpara University of Agriculture, Abia State, Nigeria \\ Email:somaliki@gmail.com,chrisdydx@yahoo.com
}

How to cite this paper: Maliki, O.S. and Chibuike, O.C. (2021) A Mathematical Model for the Control of Cholera Epidemic without Natural Recovery. Applied Mathematics, 12, 655-668. https://doi.org/10.4236/am.2021.128046

Received: November 6, 2020

Accepted: August 10, 2021

Published: August 13, 2021

Copyright (อ 2021 by author(s) and Scientific Research Publishing Inc. This work is licensed under the Creative Commons Attribution International License (CC BY 4.0).

http://creativecommons.org/licenses/by/4.0/

\section{(c) (i) Open Access}

\begin{abstract}
In this research work, we present a mathematical model for the control of cholera outbreak without natural recovery. This follows a slight modification as compared to previous cholera models for the Nigerian case. Our model incorporates treatment, water hygiene as well as environmental sanitation. The model employs a system of nonlinear ordinary differential equations, which is analyzed in detail for its stability properties. We compute the basic reproduction ratio $R_{0}$ for the various control parameters and discover that with proper combination of control measures, the spread of cholera could be minimized. Numerical simulation of the cholera model is done using MathCAD14, and the graphical profiles of the main variables are depicted. We conclude that improvement in treatment, water hygiene and the environmental sanitation is indeed effective in eradicating the cholera epidemic.
\end{abstract}

\section{Keywords}

Cholera Control Model, Differential Equations, Basic Reproduction Ratio, MathCAD Simulation

\section{Introduction}

Cholera is a contagious infectious disease that is characterized by extreme vomiting, profuse watery diarrhoea and leg pain. It has been found that transmission transpires mostly via absorption of contaminated drinking water or food. Worldwide, almost every year, there are estimated 3 - 5 million cholera cases and $100,000-130,000$ deaths due to cholera a year as of 2010 [1]. It has a very short incubation period which starts from a few hours to five days. The health of an infected person disintegrates rapidly and death may occur if treatment is not promptly given. Cholera was first discovered in the Indian subcontinent in 1817. The disease reaches all the way through Asian continent in the 1960s, getting 
into Africa in 1970 and Latin America in 1991 [2] [3]. In many parts of Africa and Asia, the disease is still endemic. Cholera is a disastrous water-borne infectious disease that is caused by the bacterium vibrio cholera. It is a very serious problem in many developing countries due to inadequate access to safe drinking water supply, improper treatment of reservoirs and improper sanitation. In 2012, WHO reported 245, 393 cholera cases and 3034 death cases across 48 countries in which 67\% cases occurred in African countries [4]. In 2005, Nigeria had 4477 cases and 174 deaths. There were reported cases of cholera in 2008 in Nigeria in which there were 429 deaths out of 6330 cases. Furthermore, 2304 cases were reported in Niger State in which 114 were death cases [5]. Also in 2009, Nigeria reported 13,691 cases and 431 deaths [6] (WHO). Tian and Wang [7] evidenced that recent years have seen a strong trend of cholera outbreak in developing countries, such as in India (2007), Iraq (2008), Congo (2008), Zimbabwe (2008-2009), Haiti (2010), Kenya (2010) and Nigeria (2010).

In Nigeria, outbreaks of the disease have been taking place with ever-increasing occurrence ever since the earliest outbreak in recent times in 1970, [8] [9]. In summary, the United Nation (UN) unit reports: "despite Nigeria's oil wealth, more than $70 \%$ of the country's 126 million people live below the poverty line and cholera outbreaks are common in poor urban areas which lack proper sanitation and clean drinking water" (UN Office for the Coordination of $\mathrm{Hu}$ manitarian Affairs Integrated Regional Information Networks (IFIN) 2005). In the last few decades, several researchers [10] [11] [12] [13] [14] have designed mathematical models to explore the transmission dynamics and control of the disease. However, in the literature, not many studies on cholera in Nigeria using bio-mathematical models are prevalent, hence, the need for this research study.

\subsection{Model Formulation}

A mathematical model for cholera transmission is developed by making a slight modification to the previous model by Sulayman Fatima et al. [15]. Our model is devoid of natural recovery, this is because from the literature we find that cholera is a fatal disease, and a large percentage of those who recover, do so because of treatment. Our model on the other hand incorporates treatment, water hygiene and environmental sanitation. The model contains five variables which are susceptible, infected, recovered, total human population, and the concentration of vibrio cholera in water. The susceptible population is generated either through birth or through immigration. They acquire infection and move to the infected class at the rate:

$$
\frac{\alpha\left(1-c_{w}\right) B}{K+B}
$$

$a=$ rate of exposure to contaminated water;

$\mathcal{c}_{W}=$ rate of compliance with water hygiene;

$K=$ concentration of vibrio cholera in water that yields $50 \%$ chance of catching cholera; 
$B=$ concentration of vibrio cholera in contaminated water.

The number of infected individuals decreases through recovery from the disease at the rate $\tau$, being recovery due to treatment. Bacterial populations in the aquatic environment grow in the water at the rate determined by the environmental factors such as temperature. The variables and parameters of the model are described in Table 1 and Table 2: A compartmental representation of our model is given in Figure 1: The model consists of the following system of nonlinear ordinary differential equations given in (2) to (5):

Table 1. Variables of the cholera model.

\begin{tabular}{cl}
\hline Symbol & \\
\hline$S(t)$ & Susceptible human population at time $t$ \\
$I(t)$ & Infected human population at time $t$ \\
$R(t)$ & Recovered human population at time $t$ \\
$B(t)$ & Concentration of vibrio cholera population at time $t$ \\
$N$ & Total population of humans \\
\hline
\end{tabular}

Table 2. Parameters of the cholera model.

\begin{tabular}{cl}
\hline Symbol & \\
\hline$\beta$ & Per capita birth rate of humans \\
$\mu$ & Per capita death rate of humans \\
$a$ & Rate of exposure to contaminated water \\
$K$ & Concentration of vibrio cholera in water \\
$\tau$ & Recovery rate due to treatment \\
$c_{w}$ & Rate of compliance with water hygiene \\
$C_{s}$ & Rate of compliance with environmental sanitation \\
$\delta$ & Cholera induced death \\
$\rho$ & Growth rate of vibrio cholera in the aquatic environment \\
$\omega$ & Loss rate of immunity by recovered individuals \\
$e$ & Contribution of each infected person to the population of vibrio cholera in the \\
\hline
\end{tabular}

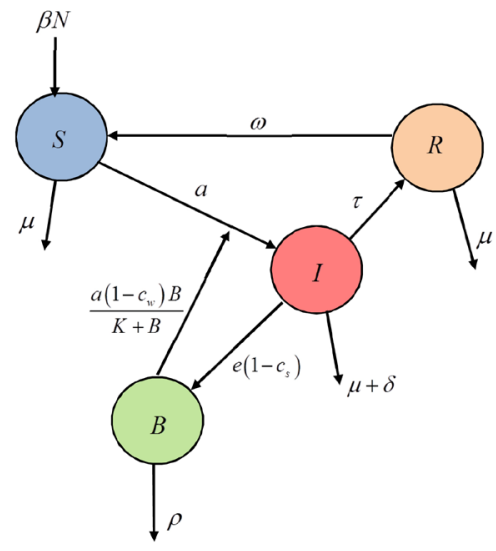

Figure 1. Compartmental model of controlled cholera transmission model without natural recovery. 


$$
\begin{gathered}
\frac{\mathrm{d} S}{\mathrm{~d} t}=\beta N-\frac{a\left(1-c_{w}\right) B}{K+B} S+\omega R-\mu S \\
\frac{\mathrm{d} I}{\mathrm{~d} t}=\frac{a\left(1-c_{w}\right) B}{K+B} S-(\tau+\mu+\delta) I \\
\frac{\mathrm{d} B}{\mathrm{~d} t}=e\left(1-c_{s}\right) I-\rho B \\
\frac{\mathrm{d} R}{\mathrm{~d} t}=\tau I-(\omega+\mu) R
\end{gathered}
$$

With

$$
N=S+I+R
$$

\subsection{Remark}

The variables and parameters of the model are presented in Table 1 and Table 2, while their respective baseline values are given in Table 3 and Table 4.

\subsection{Model Analysis}

Our Cholera model represented by the system of nonlinear differential Equations (2)-(5) is now analyzed qualitatively to obtain insights into its features, which will give a better understanding of the control features on the transmission dynamics of the cholera virus.

\subsubsection{Disease Free Equilibrium (DFE)}

The disease free equilibrium of model system (1)-(4) is obtained by setting

Table 3. Baseline values for variables of the cholera model in Nigeria as at 2016.

\begin{tabular}{ccc}
\hline Variable & Baseline value & Reference \\
\hline$S(0)$ & $170,123,640$ & Assumed \\
$I(0)$ & 100 & Assumed \\
$R(0)$ & 0 & Assumed \\
$B(0)$ & $1,000,000,000$ & Assumed \\
$N(0)$ & $170,123,740$ & CIA $(2011)$
\end{tabular}

Table 4. Baseline values for parameters of the cholera model.

\begin{tabular}{ccc}
\hline Parameter & Baseline value & Reference \\
\hline$\beta$ & $170,123,640$ & Assumed \\
$\mu$ & 100 & Assumed \\
$a=10000 \mu / \beta N$ & 0.00000289 day $^{-1}$ & Estimated \\
$K$ & $1,000,000,000$ & Codecco (2001) \\
$\tau$ & $(0-1)$ day $^{-1}$ & Assumed \\
$C_{w}$ & $(0-1)$ day $^{-1}$ & Assumed \\
$C_{s}$ & $(0-1)$ day $^{-1}$ & Assumed \\
$\delta$ & 0.00008 day $^{-1}$ & WHO (2012) \\
$\rho$ & 0.003 day $^{-1}$ & Codecco (2001) \\
$e$ & 0.003 day $^{-1}$ & Mwasa \& Tchuenche (2001)
\end{tabular}


$\frac{\mathrm{d} S}{\mathrm{~d} t}=\frac{\mathrm{d} I}{\mathrm{~d} t}=\frac{\mathrm{d} B}{\mathrm{~d} t}=\frac{\mathrm{d} R}{\mathrm{~d} t}=0$, and in the absence of disease, $I=B=0$ so that:

$$
\begin{gathered}
\beta N-\frac{a\left(1-c_{w}\right) B}{K+B} S+\omega R-\mu S=0 \\
\frac{a\left(1-c_{w}\right) B}{K+B} S-(\tau+\mu+\delta) I=0 \\
e\left(1-c_{s}\right) I-\rho B=0 \\
\tau I-(\omega+\mu) R=0
\end{gathered}
$$

Since $I=B=0$, Equation (4) gives $R=0$ and Equation (1) gives $S=\frac{\beta N}{\mu}$.

Hence DFE is

$$
\left(S^{0}, I^{0}, B^{0}, R^{0}\right)=\left(\frac{\beta N}{\mu}, 0,0,0\right)
$$

\subsubsection{Positivity of Solutions}

Since the model monitors human population, we need to show that all the state variables remain non-negative for all times.

\subsubsection{Theorem}

Let $\Omega=\left\{(S, I, R, B) \in \mathbb{R}_{+}^{4}: S(0)>0, I(0)>0, R(0)>0, B(0)>0\right\}$ then the solutions of $\{S(t), I(t), R(t), B(t)\}$ of the system Equation (2)-(5) are positive for all $t \geq 0$.

Proof: Consider the first equation of the system;

$$
\begin{gathered}
\frac{\mathrm{d} S}{\mathrm{~d} t}=\beta N-\frac{a\left(1-c_{w}\right) B}{K+B} S+\omega R-\mu S \\
\Rightarrow \frac{\mathrm{d} S}{\mathrm{~d} t} \geq \beta N-\mu S
\end{gathered}
$$

We consider the solution of

$$
\frac{\mathrm{d} S}{\mathrm{~d} t}+\mu S=\beta N
$$

Employing the integrating factor method, we obtain $S(t)=\frac{\beta N}{\mu}+C \mathrm{e}^{-\mu t} \quad(C=$ constant of integration $)$

With the initial condition $S(0)=S_{0} \Rightarrow C=S_{0}-\frac{\beta N}{\mu}$,

$$
\therefore \quad S(t)=\frac{\beta N}{\mu}+\left(S_{0}-\frac{\beta N}{\mu}\right) \mathrm{e}^{-\mu t} \geq 0
$$

We now consider the second equation;

$$
\begin{gathered}
\frac{\mathrm{d} I}{\mathrm{~d} t}=\frac{a\left(1-c_{w}\right) B}{K+B} S-(\tau+\mu+\delta) I \\
\therefore \quad \frac{\mathrm{d} I}{\mathrm{~d} t} \geq-(\tau+\mu+\delta) I
\end{gathered}
$$


Integration yields

$$
I(t) \geq I_{0} \mathrm{e}^{-(\tau+\mu+\delta) t} \geq 0
$$

We consider also the third equation

$$
\begin{gathered}
\frac{\mathrm{d} B}{\mathrm{~d} t}=e\left(1-c_{s}\right) I-\rho B \\
\therefore \quad \frac{\mathrm{d} B}{\mathrm{~d} t} \geq-\rho B \\
\Rightarrow \quad B(t) \geq B_{0} \mathrm{e}^{-\rho B t}
\end{gathered}
$$

We observe that as $t \rightarrow \infty, B(t) \rightarrow B_{0}$.

Finally from the fourth equation

$$
\begin{aligned}
& \frac{\mathrm{d} R}{\mathrm{~d} t}=\tau I-(\omega+\mu) R \\
& \frac{\mathrm{d} R}{\mathrm{~d} t} \geq-(\omega+\mu) R \\
\Rightarrow \quad & R(t) \geq R_{0} \mathrm{e}^{-(\omega+\mu) t} \geq 0
\end{aligned}
$$

\subsubsection{Invariant Region}

The system Equations (2)-(5) have solutions which are contained in the feasible region:

$$
\Omega=\left\{(S, I, R, B) \in \mathbb{R}_{+}^{4}: 0 \leq S(0), 0 \leq I(0), 0 \leq R(0), 0 \leq B(0), S+I+R \leq \frac{\beta N}{\mu}\right\}
$$

where the model makes biological sense, and can be shown to be positively invariant and globally attracting in $\mathbb{R}_{+}^{4}$ with respect to the system of ODE describing our model.

\subsection{Model Analysis}

The model system (2)-(5) is analyzed qualitatively to get insights into its dynamical features which give better understanding of the impact control strategies on the transmission dynamics of the cholera disease. First, we study the stability properties of the cholera model.

\subsubsection{Stability Analysis of the Cholera Model}

Here we consider the Jacobian matrix associated with the system of differential equations for our Cholera model

$$
J(S, I, B, R)=\left(\begin{array}{llll}
\frac{\partial f_{1}}{\partial S} & \frac{\partial f_{1}}{\partial I} & \frac{\partial f_{1}}{\partial B} & \frac{\partial f_{1}}{\partial R} \\
\frac{\partial f_{2}}{\partial S} & \frac{\partial f_{2}}{\partial I} & \frac{\partial f_{2}}{\partial B} & \frac{\partial f_{2}}{\partial R} \\
\frac{\partial f_{3}}{\partial S} & \frac{\partial f_{3}}{\partial I} & \frac{\partial f_{3}}{\partial B} & \frac{\partial f_{3}}{\partial R} \\
\frac{\partial f_{4}}{\partial S} & \frac{\partial f_{4}}{\partial I} & \frac{\partial f_{4}}{\partial B} & \frac{\partial f_{4}}{\partial R}
\end{array}\right)
$$


where

$$
\begin{gathered}
f_{1}(S, I, B, R)=\beta N-\frac{a\left(1-c_{w}\right) B}{K+B} S+\omega R-\mu S \\
f_{2}(S, I, B, R)=\frac{a\left(1-c_{w}\right) B}{K+B} S-(\tau+\mu+\delta) I \\
f_{3}(S, I, B, R)=e\left(1-c_{s}\right) I-\rho B \\
f_{4}(S, I, B, R)=\tau I-(\omega+\mu) R
\end{gathered}
$$

Computing the respective partial derivatives, we obtain;

$$
J(S, I, B, R)=\left(\begin{array}{cccc}
-\frac{a\left(1-c_{w}\right) B}{K+B}-\mu & 0 & -\frac{a K\left(1-c_{w}\right)}{(K+B)^{2}} & \omega \\
\frac{a\left(1-c_{w}\right) B}{K+B} & -(\tau+\mu+\delta) & \frac{a K\left(1-c_{w}\right)}{(K+B)^{2}} & 0 \\
0 & e\left(1-c_{s}\right) & -\rho & 0 \\
0 & \tau & 0 & -(\omega+\mu)
\end{array}\right)
$$

Evaluating the Jacobian at the disease free equilibrium state

$$
\begin{aligned}
\left(S^{0}, I^{0}, B^{0}, R^{0}\right) & =\left(\frac{\beta N}{\mu}, 0,0,0\right) \text { gives } \\
J^{*}\left(S^{0}, I^{0}, B^{0}, R^{0}\right) & =\left(\begin{array}{cccc}
-\mu & 0 & -\frac{a\left(1-c_{w}\right)}{K} & \omega \\
0 & -(\tau+\mu+\delta) & \frac{a\left(1-c_{w}\right)}{K} & 0 \\
0 & e\left(1-c_{s}\right) & -\rho & 0 \\
0 & \tau & 0 & -(\omega+\mu)
\end{array}\right)
\end{aligned}
$$

The eigenvalues of $J^{*}$ are given by

$$
\left|J^{*}-\lambda I\right|=0
$$

Giving the characteristic equation;

$$
\lambda^{4}+A \lambda^{3}+B \lambda^{2}+C \lambda+D=0
$$

where

$$
A=3 \mu+\rho+\tau+\omega+\delta
$$

$B=\frac{3 K \mu^{2}-a e+a e c_{s}+a e c_{w}+3 K \mu \rho+2 K \mu \tau+K \rho \tau+2 K \mu \delta+2 K \mu \omega+K \rho \delta+K \rho \omega+K \tau \omega+K \omega \delta-a e c_{s} c_{w}}{K}$

$$
\begin{aligned}
& K \mu^{3}+3 K \mu^{2} \rho+K \mu^{2} \tau+K \mu^{2} \omega+K \mu^{2} \delta-2 a e \mu-a e \omega+2 a e \mu c_{s}+2 a e \mu c_{w}+a e \omega c_{s}+a e \omega c_{w} \\
& C=\frac{+2 K \mu \rho \tau+2 K \mu \rho \omega+2 K \mu \rho \delta+K \mu \tau \omega+K \rho \tau \omega+K \mu \omega \delta+K \rho \omega \delta-2 a e \mu c_{s} c_{w}-a e \omega c_{s} c_{w}}{K} \\
& K \mu^{3} \rho-a \mu^{2} e-a \mu \omega e+a \mu^{2} c_{s} e+a \mu^{2} c_{w} e+K \mu^{2} \rho \tau+K \mu^{2} \rho \omega+K \mu^{2} \rho \delta \\
& D=\frac{+a \mu \omega c_{s} e+K \mu \rho \tau \omega+K \mu \rho \omega \delta-a \mu^{2} c_{s} c_{w} e-a \mu \omega c_{s} c_{w} e}{K}
\end{aligned}
$$




\subsubsection{Remark}

We have asymptotic stability of the disease free equilibrium provide all the roots of the quartic Equation (15) have negative real parts.

\subsection{The Basic Reproduction Ratio, $R_{0}$}

The basic reproduction ratio (or number) denoted by $R_{0}$ is the average number of secondary infections caused by an infectious individual during his or her entire period of infectiousness (Diekmann et al. [16]). The basic reproduction ratio is an important non-dimensional quantity in epidemiology because it sets the threshold in the study of any given disease, both for predicting its outbreak and for evaluating its control strategies.

\subsubsection{The Next Generation Matrix Approach}

In general $R_{0}$ is found through the study and computation of the eigenvalues of the Jacobianmatrix at the disease- or infectious-free equilibrium. Diekmann et al. [16] follow a different approach which is the next generation matrix method. This procedure converts a system of ordinary differential equations of a model of infectious disease dynamics to an operator (or matrix) that translate from one generation of infectious individuals to the next. The basic reproductive number is then defined as the spectral radius (dominant eigenvalue) of this operator.

\subsubsection{Remark}

We deduce that whether a disease becomes persistent or dies out in a community is totally dependent on the value of the basic reproduction ratio, $R_{0}$. Furthermore, stability of equilibria can be analyzed using $R_{0}$. If $R_{0}<1$, it means that every infectious individual will cause less than one secondary infection and hence the disease will die out and when $R_{0}>1$, every infectious individual will cause more than one secondary infection and hence the disease will invade the population.

A large value of $R_{0}$ may indicate the possibility of a major epidemic. For the case of a model with a single infected class, $R_{0}$ is simply the product of the infection rate and the mean duration of the infection.

\subsubsection{The Basic Reproduction Ratio for the Cholera Model}

The basic reproduction ratio is obtained by taking the dominant eigenvalue of

$$
F V^{-1}=\left[\frac{\partial F_{i}\left(E_{0}\right)}{\partial x_{j}}\right]\left[\frac{\partial V_{i}\left(E_{0}\right)}{\partial x_{j}}\right]^{-1}
$$

where $F_{i}$ is the rate of appearance of new infection in compartment $i, V_{i}$ is the transfer of infections from one compartment $i$ to another and $E_{0}$ is the disease-free equilibrium. From the system of differential equations we identify the equations with infectious classes as

$$
\frac{\mathrm{d} I}{\mathrm{~d} t}=\frac{a\left(1-c_{w}\right) B}{K+B} S-(\tau+\mu+\delta) I
$$


and

$$
\frac{\mathrm{d} B}{\mathrm{~d} t}=e\left(1-c_{\mathrm{s}}\right) I-\rho B
$$

We thus have

$$
F_{i}=\left[\begin{array}{c}
\frac{a\left(1-c_{w}\right) B S}{K+B} \\
0
\end{array}\right]
$$

Hence

$$
F=\left[\begin{array}{ll}
\frac{\partial F_{1}}{\partial I} & \frac{\partial F_{1}}{\partial B} \\
\frac{\partial F_{2}}{\partial I} & \frac{\partial F_{2}}{\partial B}
\end{array}\right]=\left[\begin{array}{cc}
0 & \frac{a\left(1-c_{w}\right) S^{0}}{K} \\
0 & 0
\end{array}\right]
$$

Similarly

$$
V_{i}=\left[\begin{array}{c}
(\tau+\mu+\delta) I \\
-e\left(1-c_{s}\right) I+\rho B
\end{array}\right]
$$

Hence

$$
\begin{aligned}
V & =\left[\begin{array}{ll}
\frac{\partial V_{1}}{\partial I} & \frac{\partial V_{1}}{\partial B} \\
\frac{\partial V_{2}}{\partial I} & \frac{\partial V_{2}}{\partial B}
\end{array}\right]=\left[\begin{array}{cc}
(\tau+\mu+\delta) & 0 \\
-e\left(1-c_{s}\right) & \rho
\end{array}\right] \\
\Rightarrow \quad V^{-1} & =\left[\begin{array}{ll}
(\tau+\mu+\delta) & 0 \\
-e\left(1-c_{s}\right) & \rho
\end{array}\right]^{-1}=\left[\begin{array}{cc}
\frac{1}{(\tau+\mu+\delta)} & 0 \\
\frac{e\left(1-c_{s}\right)}{\rho(\tau+\mu+\delta)} & \frac{1}{\rho}
\end{array}\right]
\end{aligned}
$$

$\therefore \quad F V^{-1}=\left[\begin{array}{cc}0 & \frac{a\left(1-c_{w}\right) S^{0}}{K} \\ 0 & 0\end{array}\right]\left[\begin{array}{cc}\frac{1}{(\tau+\mu+\delta)} & 0 \\ \frac{e\left(1-c_{s}\right)}{\rho(\tau+\mu+\delta)} & \frac{1}{\rho}\end{array}\right]=\left[\begin{array}{cc}\frac{a e\left(1-c_{w}\right)\left(1-c_{s}\right) S^{0}}{K(\tau+\mu+\delta)} & \frac{a\left(1-c_{w}\right) S^{0}}{K \rho} \\ 0 & 0\end{array}\right]$ where $S^{0}=\frac{\beta N}{\mu}$. The eigenvalues of $F V^{-1}$ are given by

$$
\left|F V^{-1}-\lambda I\right|=0
$$

or

$$
\left|\left[\begin{array}{cc}
\frac{a e\left(1-c_{w}\right)\left(1-c_{s}\right) S^{0}}{K(\tau+\mu+\delta)} & \frac{a\left(1-c_{w}\right) S^{0}}{K \rho} \\
0 & 0
\end{array}\right]-\lambda\left[\begin{array}{ll}
1 & 0 \\
0 & 1
\end{array}\right]\right|=0
$$

Giving the characteristic equation;

$$
\frac{K \rho(\tau+\mu+\delta) \lambda^{2}-S^{0} a e\left(1-c_{w}\right)\left(1-c_{s}\right) \lambda}{K \rho(\tau+\mu+\delta)}=0
$$


i.e.,

$$
\begin{gathered}
K \rho(\tau+\mu+\delta) \lambda^{2}-S^{0} a e\left(1-c_{w}\right)\left(1-c_{s}\right) \lambda=0 \\
\therefore \quad \lambda\left[K \rho(\tau+\mu+\delta) \lambda-S^{0} a e\left(1-c_{w}\right)\left(1-c_{s}\right)\right]=0 \\
\quad \Rightarrow \quad \lambda=0, \lambda=\frac{a e\left(1-c_{w}\right)\left(1-c_{s}\right) S^{0}}{K \rho(\tau+\mu+\delta)}
\end{gathered}
$$

Hence

$$
R_{0}=\frac{a e\left(1-c_{w}\right)\left(1-c_{s}\right) S^{0}}{K \rho(\tau+\mu+\delta)}
$$

Substituting $S^{0}=\frac{\beta N}{\mu}$.

$$
\Rightarrow \quad R_{0}=\frac{\beta N a e\left(1-c_{w}\right)\left(1-c_{s}\right)}{K \rho \mu(\tau+\mu+\delta)}
$$

\subsection{Control Measures with Various Strategies}

As shown in Equations (2) to (5), our model incorporates three new control measures namely treatment, water hygiene and environmental sanitation. In this study, it is of main interest to see the effect of control measures at different levels of control strategies. Using the baseline values for variables and parameters as in Table 3 and Table 4 we compute the effect of control measures $C_{w}, C_{s}$ and $\tau$ at seven different control strategies as explained below. The seven control strategies are:

1) No control: This is a situation where there is no control $\left(\tau=C_{w}=C_{s}=0\right)$.

In this case

$$
R_{0}=\frac{\beta N a e}{K \rho \mu(\mu+\delta)}
$$

2) Low without treatment: Low efficiency level of the rate of compliance with water hygiene and rate of compliance with environmental sanitation at $25 \%$ $\left(\tau=0, C_{w}=C_{s}=0.25\right)$.

$$
\Rightarrow R_{0}=\frac{9 \beta N a e}{16 K \rho \mu(\mu+\delta)}
$$

3) Moderate without treatment: Moderate effectiveness level of rate of compliance with water hygiene and rate of compliance with environmental sanitation at $50 \%\left(\tau=0, C_{w}=C_{s}=0.50\right)$.

$$
\Rightarrow R_{0}=\frac{\beta N a e}{4 K \rho \mu(\mu+\delta)}
$$

4) High without treatment: High effectiveness level of rate of compliance with water hygiene and rate of compliance with environmental sanitation at $75 \%$ $\left(\tau=0, C_{w}=C_{s}=0.75\right)$.

$$
\Rightarrow R_{0}=\frac{\beta N a e}{16 K \rho \mu(\mu+\delta)}
$$


5) Low universal: Low level of all the three control strategies at $25 \%$ $\left(\tau=C_{w}=C_{s}=0.25\right)$.

$$
\Rightarrow R_{0}=\frac{9 \beta N a e}{4 K \rho \mu(1+4 \mu+4 \delta)}
$$

6) Moderate universal: Moderate level of all the three control strategies at $50 \%\left(\tau=C_{w}=C_{s}=0.50\right)$.

$$
\Rightarrow R_{0}=\frac{\beta N a e}{2 K \rho \mu(1+2 \mu+2 \delta)}
$$

7) High universal: High level of all the three control strategies at $75 \%$ $\left(\tau=C_{w}=C_{s}=0.75\right)$.

$$
\Rightarrow R_{0}=\frac{\beta N a e}{4 K \rho \mu(3+4 \mu+4 \delta)}
$$

\subsection{MathCAD Simulation}

$\beta:=0.000107, \quad \mathrm{a}:=0.00000289, \mathrm{~K}:=10^{6}, \tau:=0.2, \rho:=0.33, \omega:=0.003$, $\mu:=0.0000526, \delta:=0.2, \Omega:=740, \mathrm{c}_{\mathrm{w}}:=0.25, \mathrm{c}_{\mathrm{s}}:=0.25, \mathrm{e}:=10$.

Define a function that determines a vector of derivative values at any solution point $(t, Y)$ :

$$
\mathrm{D}(\mathrm{t}, \mathrm{Y}):=\left[\begin{array}{c}
\beta \cdot \Omega-\frac{\mathrm{a} \cdot\left(1-\mathrm{c}_{\mathrm{w}}\right) \cdot \mathrm{Y}_{2} \cdot \mathrm{Y}_{0}}{\mathrm{~K}+\mathrm{Y}_{2}}+\omega \cdot \mathrm{Y}_{3}-\mu \cdot \mathrm{Y}_{0} \\
\frac{\mathrm{a} \cdot\left(1-\mathrm{c}_{\mathrm{w}}\right) \cdot \mathrm{Y}_{2}}{\mathrm{~K}+\mathrm{Y}_{2}} \cdot \mathrm{Y}_{0}-(\tau+\mu+\delta) \cdot \mathrm{Y}_{1} \\
\mathrm{e} \cdot\left(1-\mathrm{c}_{\mathrm{s}}\right) \cdot \mathrm{Y}_{1}-\rho \cdot \mathrm{Y}_{2} \\
\tau \cdot \mathrm{Y}_{1}-(\omega+\mu) \cdot \mathrm{Y}_{3}
\end{array}\right]
$$

Define additional arguments for the ODE solver:

$\mathrm{t} 0:=0:$ Initial value of independent variable.

Y0: $=\left(\begin{array}{c}640 \\ 100 \\ 10^{6} \\ 0\end{array}\right):$ Vector of initial function values.

$\mathrm{t} 1:=10:$ Final value of independent variable.

num: $=1 \times 10^{3}$ : Number of solution values on $[\mathrm{t} 0, \mathrm{t} 1]$.

$\mathrm{t}:=\mathrm{S}^{\langle 0\rangle}$ : Independent variable values.

$\mathrm{S}:=\mathrm{S} 1^{\langle 1\rangle}:$ First solution function values.

$\mathrm{I}:=\mathrm{S1}^{\langle 2\rangle}$ : Second solution function values.

$\mathrm{B}:=\mathrm{S}^{\langle 3\rangle}$ : Third solution function values.

$\mathrm{R}:=\mathrm{S}^{\langle 4\rangle}:$ Fourth solution function values.

The table of values for the matrix $S$ is given in Figure 2.

\subsection{Results and Discussion}

The graphical profiles in Figures 3(a)-(d) depict respectively the variation of the 


\begin{tabular}{|r|r|r|r|r|r|}
\hline & \multicolumn{1}{|c|}{0} & \multicolumn{1}{c|}{1} & \multicolumn{1}{c|}{2} & \multicolumn{1}{c|}{3} & \multicolumn{1}{c|}{4} \\
\hline 0 & 0 & 640 & 100 & $1 \cdot 10^{6}$ & 0 \\
\hline 1 & 0.1 & 640.005 & 96.079 & $9.676 \cdot 10^{5}$ & 1.96 \\
\hline 2 & 0.2 & 640.01 & 92.311 & $9.362 \cdot 10^{5}$ & 3.843 \\
\hline 3 & 0.3 & 640.016 & 88.691 & $9.058 \cdot 10^{5}$ & 5.651 \\
\hline 4 & 0.4 & 640.022 & 85.213 & $8.764 \cdot 10^{5}$ & 7.388 \\
\hline 5 & 0.5 & 640.029 & 81.871 & $8.48 \cdot 10^{5}$ & 9.056 \\
\hline 6 & 0.6 & 640.037 & 78.661 & $8.205 \cdot 10^{5}$ & 10.658 \\
\hline 7 & 0.7 & 640.045 & 75.576 & $7.939 \cdot 10^{5}$ & 12.197 \\
\hline 8 & 0.8 & 640.053 & 72.612 & $7.681 \cdot 10^{5}$ & 13.675 \\
\hline 9 & 0.9 & 640.062 & 69.765 & $7.432 \cdot 10^{5}$ & 15.094 \\
\hline 10 & 1 & 640.071 & 67.029 & $7.191 \cdot 10^{5}$ & 16.457 \\
\hline 11 & 1.1 & 640.081 & 64.4 & $6.957 \cdot 10^{5}$ & 17.766 \\
\hline 12 & 1.2 & 640.091 & 61.875 & $6.732 \cdot 10^{5}$ & 19.023 \\
\hline 13 & 1.3 & 640.101 & 59.449 & $6.513 \cdot 10^{5}$ & 20.23 \\
\hline 14 & 1.4 & 640.112 & 57.117 & $6.302 \cdot 10^{5}$ & 21.389 \\
\hline 15 & 1.5 & 640.123 & 54.878 & $6.097 \cdot 10^{5}$ & $\ldots$ \\
\hline
\end{tabular}

Figure 2. Cross section of solution matrix.

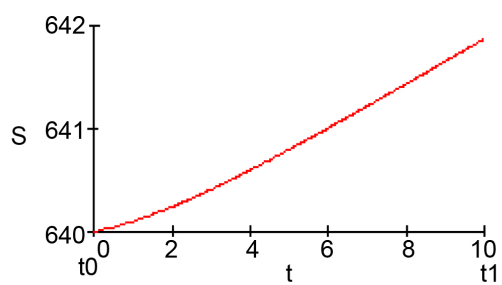

(a)

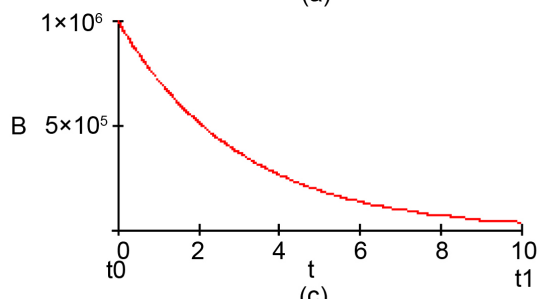

(c)

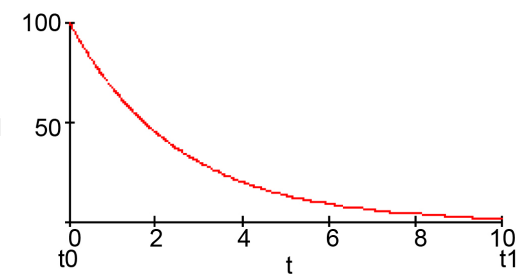

(b)

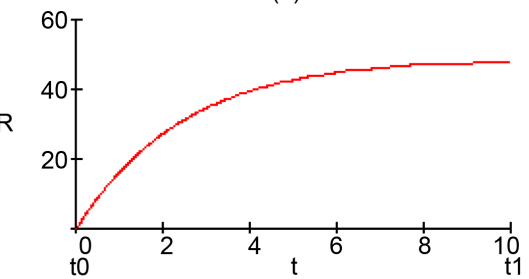

(d)

Figure 3. (a) Variation of susceptible population with time. (b) Variation of infective population with time. (c) Variation of concentration of vibriocholera population with time. (d) Variation of recovered human population with time.

intrinsic variables of the our model, namely the susceptible class $S$, the infected class $I$, the concentration of vibrio cholera population $B$, and the removed class $R$. The variation of the susceptible population shows a sharp rise from the initial value over time. However, the infective population deceases exponentially over the same time period, while the concentration of vibrio cholera decreases exponentially to zero due, to intervention measures. This in turn enables an increase in the recovered population, which reaches a saturation level over time.

\subsection{Conclusion}

An $S I R-B$ model was modified and analyzed. The model incorporates treatment, water hygiene as well as environmental sanitation. The model employs a system of nonlinear ordinary differential equations, which is studied in detail for its sta- 
bility properties. The basic reproduction ratio $R_{0}$ was computed for the various control parameters and it is discovered that with proper combination of control measures, the spread of cholera could be minimized. Numerical simulation of the cholera model was performed using the numerical software MathCAD14, and the graphical profiles of the intrinsic variables are depicted. We conclude that improvement in treatment, water hygiene and the environmental sanitation is indeed effective in eradicating the cholera epidemic.

\section{Conflicts of Interest}

The authors declare no conflicts of interest regarding the publication of this paper.

\section{References}

[1] WHO (World Health Organization) (2013) Cholera 2012. The Weekly Epidemiological Record, 88, 321-336.

[2] Codecco, C.T. (2001) Endemic and Epidemic Dynamics of Cholera: The Role of the Aquatic Reservoir. BMC Infectious Diseases, 1, Article No. 1. https://doi.org/10.1186/1471-2334-1-1

[3] Wearing, H.J., Rohani, P. and Keeling, M.J. (2005) Appropriate Models for Management of Infectious Disease. PLOS Medicine, 2, e174.

https://doi.org/10.1371/journal.pmed.0020174

[4] WHO (World Health Organization) (2013) Media Center: Cholera. https://www.who.int/news-room/fact-sheets/detail/cholera

[5] NBS (National Bureau of Statistics) (2009) Social Statistics in Nigeria. https://www.nigerianstat.gov.ng/

[6] WHO (World Health Organization) (2012) Global Task on Cholera Control. Cholera Country Profile: Nigeria.

https://www.who.int/cholera/publications/final\%20outbreak\%20booklet\%20260105 -OMS.pdf

[7] Tian, J.P. and Wang, J. (2011) Global Stability for Cholera Epidemic Models. Mathematical Biosciences, 232, 341. https://doi.org/10.1016/j.mbs.2011.04.001

[8] Epstein, P.R. (1993) Algal Blooms in the Spread and Persistence of Cholera. Biosystems, 31, 209-221. https://doi.org/10.1016/0303-2647(93)90050-M

[9] Lawoyin, T.O., Ogunbode, W.A., Olumide, E.A.A. and Onadeko, M.O. (2004) Outbreak of Cholera in Ibadan, Nigeria. European Journal of Epidemiology, 15, 365-368. https://doi.org/10.1023/A:1007547117763

[10] Capasso, V. and Paveri-Fontana, S.L. (1979) A Mathematical Model for the 1973 Cholera Epidemic in the European Mediterranean Region. Revue d'Epidémiologie et de Santé Publique, 27, 121-132.

[11] Pascual, M., Bouma, M.J. and Dobson, A.P. (2002) Cholera and Climate: Revisiting the Quantitative Evidence. Microbes and Infection, 4, 237-245.

https://doi.org/10.1016/S1286-4579(01)01533-7

[12] Hartley, D.M., Morris, J.B. and Smith, D.L. (2006) Hyperinfectivity: A Critical Element in the Ability of $V$. cholerae to Cause Epidemics. PLOS Medicine, 3, e7. https://doi.org/10.1371/journal.pmed.0030007

[13] Tien, J.H. and Earn, D.J.D. (2010) Multiple Transmission Pathways and Disease 
Dynamics in a Waterborne Pathogen Model. Bulletin of Mathematical Biology, 72, 1506-1533. https://doi.org/10.1007/s11538-010-9507-6

[14] Misra, A.K. and Singh, V. (2012) A Delay Mathematical Model for the Spread and Control of Water Borne Diseases. Journal of Theoretical Biology, 301, 49-56. https://doi.org/10.1016/j.jtbi.2012.02.006

[15] Fatima, S., Krishnarajah, I., Jaffar, M.Z.A.M. and Adam, M.B. (2014) A Mathematical Model for the Control of Cholera in Nigeria. Research Journal of Environmental and Earth Sciences, 6, 321-325. https://doi.org/10.19026/rjees.6.5776

[16] Diekman, O., Heesterbeek, J.A.P. and Metz, J.A.P. (1990) On the Definition and Computation of the Basic Reproduction Ratio in the Model of Infectious Disease in Heterogeneous Populations. Journal of Mathematical Biology, 2, 265-382. 